\title{
Further Cooling Improvement by Use of Chamfers in Front/top of Electronic Components
}

\author{
Nemdili SALEHA, NemDili FAdÉla, AzZi AbBÈs \\ Department of Genie maritime Faculty of Genie Mécanique, Laboratoire Aero-Hydrodynamique \\ Navale, LAHN Usto-MB University BP1505 El-Mnnaour,3100,Oran, ALGERIA
}

\begin{abstract}
This paper presents a Computational Fluid Dynamic computation based on Reynolds Averaged Navier-Stokes Equations (RANS) and the Shear Stress Transport (SST) turbulence model. The targeted application is the cooling process of an electronic component, which is represented by a heated wall-mounted cube exposed to an impinging jet in cross flow. In a previously published study, it was shown that adding chamfers on top of the cube, the flow structure is radically changed in favor of a significant improvement in cooling efficiency. In an attempt to determine which of the four faces has the greatest contribution to improving cooling, this study presents a detailed numerical investigation for five different cases. Namely, a base cube without chamfer, a cube with four chamfers on its upper part, a cube with a chamfer on the front face, a cube with chamfer on the back face and finally a cube with chamfers on the side faces. For the same $\mathrm{ReH}=3410$ cross-flow Reynolds number, three levels of incident jets are tested as well as a jet-free case, which will serve as a reference case for comparison. Comparing the different cases, it has been found that the case of a chamfer on the front face is the most efficient and therefore contributes effectively to the improvement of the cooling efficiency
\end{abstract}

Keywords: Wall mounted cube, impinging jet, SST turbulence model, jet in cross flow, cooling

Received: April 25, 2021. Revised: July 2, 2021. Accepted: July 23, 2021. Published: August 10, 2021.

\section{Introduction}

An introduction to efficient cooling design and its relation to electronic reliability, reviewing previous works [1-13] and providing motivation for this study of adding chamfers on the top of electronic components, are given in the previous study [11] and will not be repeated here. In the mentioned paper, a Computational Fluid Dynamic (CFD) study of an impinging jet in cross-flow configuration on a heated wall-mounted cube is carried out. Figure 1 shows a sketch of the computational domain used in [11], and will be reconsidered in the present study. The geometrical configuration mimics an electronic component simultaneously cooled by a transverse flow and an impacting jet. For a constant cross flow Reynolds number $\left(R e_{H}=3410\right)$, three levels of impinging jets are computed as well as a case without impinging jet that served as baseline case for comparison. Then in order to improve the cooling efficiency, the sharp angles of the upper face of the cube are transformed by adding chamfers. Three chamfer heights are considered, namely 1, 2 and $4 \mathrm{~mm}$. In terms of percentage, and taking into account the cube height $(h=15 \mathrm{~mm})$, the three cases corresponds to $6.67 \%, 13.33 \%$ and $26.67 \%$ respectively.
Limited to the highest Reynolds number ratio $R e_{j} / R e_{H}=1.5$, it was found that the global heat transfer exchanged between the cube and the external flow is increased by about $3 \%$ for 1 and $2 \mathrm{~mm}$ chamfers and by $26 \%$ for $4 \mathrm{~mm}$ chamfer. In order to analyse the effect of each part of the chamfers, the present study focuses on three cases. The first one have a chamfer in the front face (Figure 2.c), the second case have a chamfer on both side faces (Figure 2.d), while the third case has a chamfer at the rear face (Figure 2.e). For comparison purposes two other cases are also considered, namely the regular case without chamfer (Figure 2.a) and the case with full chamfer on the upper face (Figure 2.b). Figure 2 shows the geometric shapes of the five studied cases. For the record, the regular case is almost identical to that studied experimentally by Masip et al. [6] where the main Reynolds number is fixed at 3410 and the jet Reynolds number at 5115. The main Reynolds number is based on the channel bulk velocity $\left(U_{i n f}=1.705 \mathrm{~m} / \mathrm{s}\right)$ 
and the channel height $(H=2 h)$, while the jet Reynolds number $\left(R e_{j}\right)$ is based on the bulk velocity $U_{j}(6.393 \mathrm{~m} / \mathrm{s})$ and the diameter of the orifice $D(12 \mathrm{~mm})$. The test cases will be noted hereafter as follow: F15, FC15, FF15, FR15 and FS15, for the regular cube, the cube with full chamfer, the cube with chamfer on the front face, the cube with chamfer on the rear face and the cube with chamfer on both side faces respectively. The chamfer height is $2 \mathrm{~mm}$ while the height of the cube is 15 $\mathrm{mm}$.

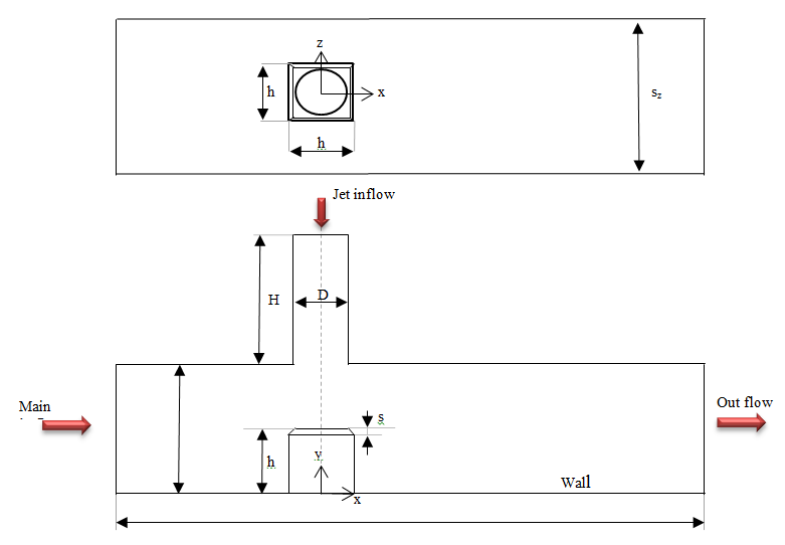

Fig. 1 Computational domain, boundary conditions and local coordinate system.
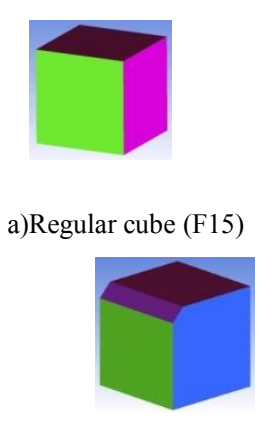

d) FF15

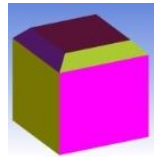

b) FC15

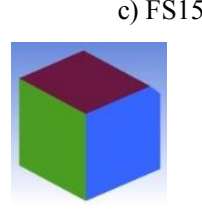

e) FR15
Fig. 2 Sketch of the modified cases

\section{Flow configuration and computational details}

As mentioned previously, the geometric details and the calculation parameters are similar to those of the experimental work of Masip et al. [6]. Thus, the calculation domain is a rectangular channel with a cube $(\mathrm{h}=15 \mathrm{~mm})$ in the middle of the bottom wall. The channel has two inputs; a left-to-right transverse flow and a downward-directed circular impact jet, as shown in Figure 1. The impact jet has a diameter of $12 \mathrm{~mm}$. In order to obtain realistic boundary conditions, a $30 \mathrm{~mm}$ long cylindrical pipe is added before the injection hole. The geometric details, properties and boundary conditions are summarized in Table 1. The boundary conditions are used as follow: the top boundary including inside the hole injection, the bottom walls including the cube and the side walls have no-slip conditions. The outflow conditions are set to zerogradient condition and finally the same temperature $20^{\circ} \mathrm{C}$ is used for the channel flow and the impinging jet. All walls boundaries are maintained at constant temperature, $60^{\circ} \mathrm{C}$ for the cube and $20^{\circ} \mathrm{C}$ for the remaining walls.

At the inlet of computational domain, the bulk velocity is established according to the Reynolds number of 3410, which corresponds to $1.705 \mathrm{~m} / \mathrm{s}$. The turbulence intensity is assigned a value of $5 \%$ and the turbulence dissipation is calculated based on a turbulent viscosity equal 10 times the laminar viscosity. At the inlet of the cylindrical tube (hole injection), the velocity is calculated according to the number of reciprocal Reynolds $R e_{j}$ (see Table 1), while the turbulent quantities are adjusted identically to the main flow.

Table 1 Geometric and flow details

\begin{tabular}{|lcc|}
\hline Jet diameter & $D$ & $12 \mathrm{~mm}$ \\
\hline Component height & $h$ & $15 \mathrm{~mm}$ \\
\hline Channel height & $H$ & $2 h=30 \mathrm{~mm}$ \\
\hline Chamfer height & $s$ & $2 \mathrm{~mm}$ \\
\hline Channel bulk velocity & $U_{\text {inf }}$ & $1.705 \mathrm{~m} / \mathrm{s}$ \\
\hline Jet bulk velocity & $U_{j}$ & $6.393 \mathrm{~m} / \mathrm{s}$ \\
\hline Computational domain length & $S_{x}$ & $1000 \mathrm{~mm}$ \\
\hline Computational domain width & $S_{z}$ & $150 \mathrm{~mm}$ \\
\hline Distance from inlet to the cube & $S_{0}$ & $300 \mathrm{~mm}$ \\
\hline $\begin{array}{l}\text { Reynolds number based on the channel height and channel } \\
\text { bulk velocity, } R e_{H} \quad 3410\end{array}$ \\
\hline $\begin{array}{l}\text { Reynolds number based on the jet diameter and jet bulk } \\
\text { velocity, }\end{array}$ \\
\hline
\end{tabular}

\section{Numerical details}

The current simulations were conducted using the ANSYSCFX package. In the solver module, the solution of the Reynolds Averaged Navier-Stokes (RANS) and energy equations is acquired using the finite volume method with a body-fitted hexahedral unstructured grid. A co-located layout is employed in which the pressure, turbulence, and velocity unknowns share the same location. The momentum and continuity equations are coupled through a pressure correction scheme.

In the present simulation, convection terms are discretized with a second order scheme except near discontinuities, where it decreases to first order to preserve boundedness. The turbulence closure is attained by use of the well-known Shear Stress Transport (SST) k-omega based model proposed by Menter et al., [8]. This model is known to provide a good compromise by combining the k-omega model of Wilcox in the near wall region and the high Reynolds k-eps model in the outer region. The use of the two models is realized via a blending function, which switches from one to zero depending on the geometrical position of the integration point. Detailed 
explanation of the model formulation and test case validations can be found in specific literature of Menter's group [8].

After many tests and according to the best compromise between precision and available computational facilities a grid of about two million structured hexahedral cells was adopted for all cases. The grid was checked at posteriori by plotting the $y+$ contours which is maintained less than unity for the first grid point away from all viscous walls. The grid points are also well stretched near the walls and in the vicinity of the cube. An illustration of the mesh quality is given in Figure 3, which shows same zooming parts near the cube, especially the $O$ grid strategy in order to avoid singular points.

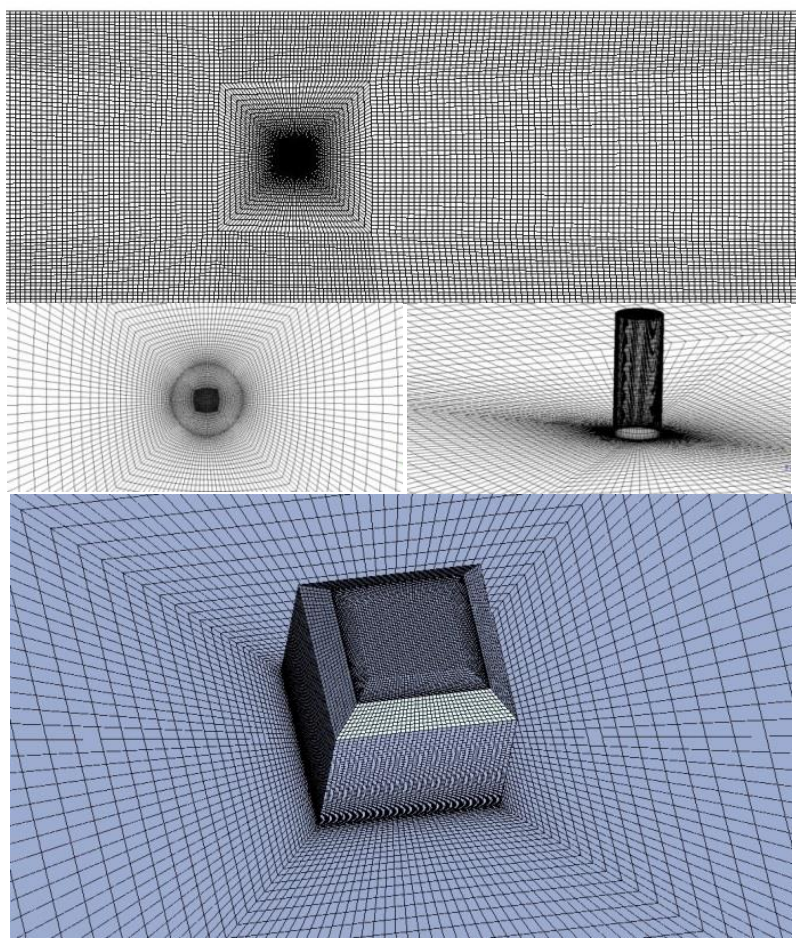

Fig. 3 Computational mesh.

To build the SST model, the Wilcox model is multiplied by a blending function $F_{1}$ and a transformed version of the $k-\varepsilon$ model by a function $\left(1-F_{1}\right) \cdot F_{1}$ is equal to one near the solid walls and decreases to a value of zero outside the boundary layer. At the boundary layer edge and outside the boundary layer, the standard $k$ - $\varepsilon$ model is therefore recovered. Then the corresponding $k$ and $\omega$ equations are added to give the new model formula given by Eqs. (1) and (2).

$$
\begin{aligned}
& \frac{\partial(\rho k)}{\partial t}+\frac{\partial\left(\rho U_{j} k\right)}{\partial x_{j}}=\frac{\partial}{\partial x_{j}}\left[\left(\mu+\frac{\mu_{t}}{\sigma_{k 3}}\right) \frac{\partial k}{\partial x_{j}}\right]+\widetilde{P}_{k}-\beta^{*} \rho k \omega+P_{k b}(1) \\
& \frac{\partial(\rho \omega)}{\partial t}+\frac{\partial\left(\rho U_{j} \omega\right)}{\partial x_{j}}=\frac{\partial}{\partial x_{j}}\left[\left(\mu+\frac{\mu_{t}}{\sigma_{\omega 3}}\right) \frac{\partial \omega}{\partial x_{j}}\right]+\left(1-F_{1}\right) 2 \rho \frac{1}{\sigma_{\omega 2} \omega} \frac{\partial k}{\partial x_{j}} \frac{\partial \omega}{\partial x_{j}}+\alpha_{3} \frac{\omega}{k} P_{k}-\beta_{3} \rho \omega^{2}
\end{aligned}
$$

The coefficients of the new model are a linear combination of the corresponding coefficients of the two previous models:

$$
\varphi=F_{1} \varphi_{1}+\left(1-F_{1}\right) \varphi_{2}
$$

All coefficients are listed here:

$$
\sigma_{k_{1}}=2.0 ; \sigma_{\omega_{1}}=2.0 ; \alpha_{1}=5 / 9 ; \beta_{1}=0.075 ; \beta^{*}=0.09 \sigma_{k_{2}}=1.0
$$

$; \sigma_{\omega_{2}}=1 / 0.856 ; \alpha_{2}=0.44 ; \beta_{2}=0.0828$

\section{Results and discussion 4.1. Regular cube and various Reynolds number ratios}

Figure 4 illustrate the streamlines in the XY plane at $\mathrm{z} / \mathrm{h}=0$ for the four cases. In the case of the high Reynolds ratio (1.5) the jet also impinges the top cube surface and additionally there is a small part of it that sweeps along the cube front face. As described by preceding investigations this part of jet sweeping along the cube front face can improve significantly the cooling of the cube. In a preliminary experiment of Flikweert [9], he showed that the maximum cooling effect of the cube was attained when the jet axis was moved upstream from the cube center by a small distance. This position increases the part of the impingement jet that sweeps along the front face.
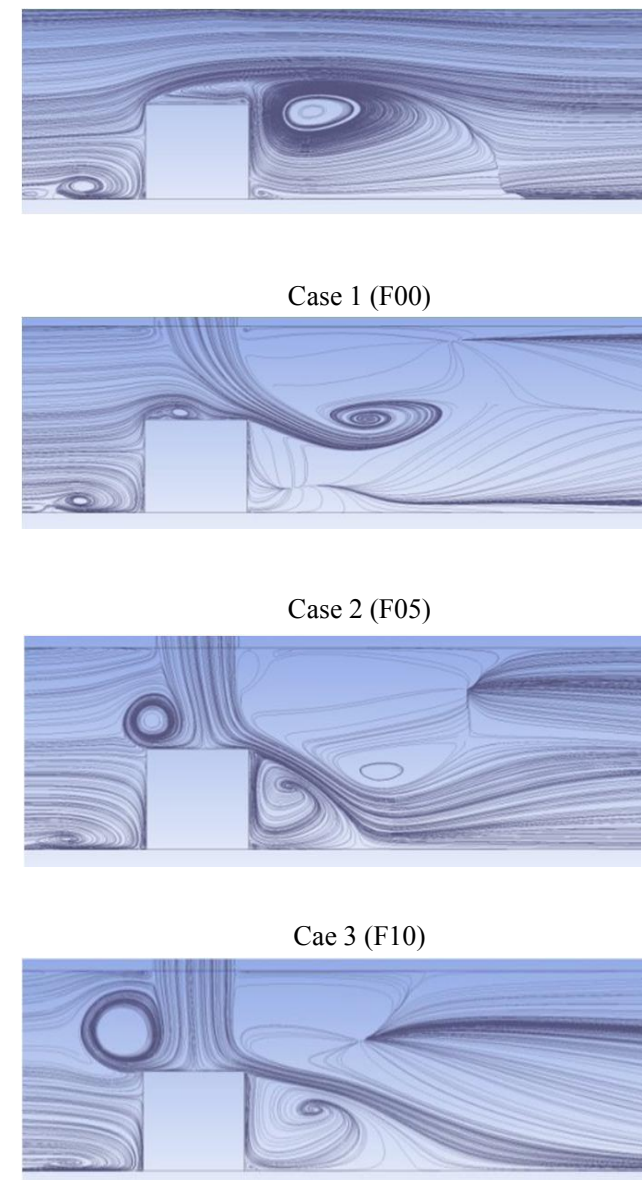

Case4 (F15)

Fig. 4 Streamlines in the $X Y$ plane at $z / h=0$ for $R e_{H}=3410$. 
WSEAS TRANSACTIONS on HEAT and MASS TRANSFER DOI: 10.37394/232012.2021.16.14

Figure 5 shows the streamline in $\mathrm{YZ}$ plane at $\mathrm{x} / \mathrm{h}=0$. The streamlines are colored by the temperature and highlight clearly the bubbles at the side faces of the cube. The jet flow decelerates at the top of the cube and then turns sharply to form a radial wall jet. At the corner, the flow separates and reattaches downstream creating a recirculation bubble that has important effect on the wall heat flux.
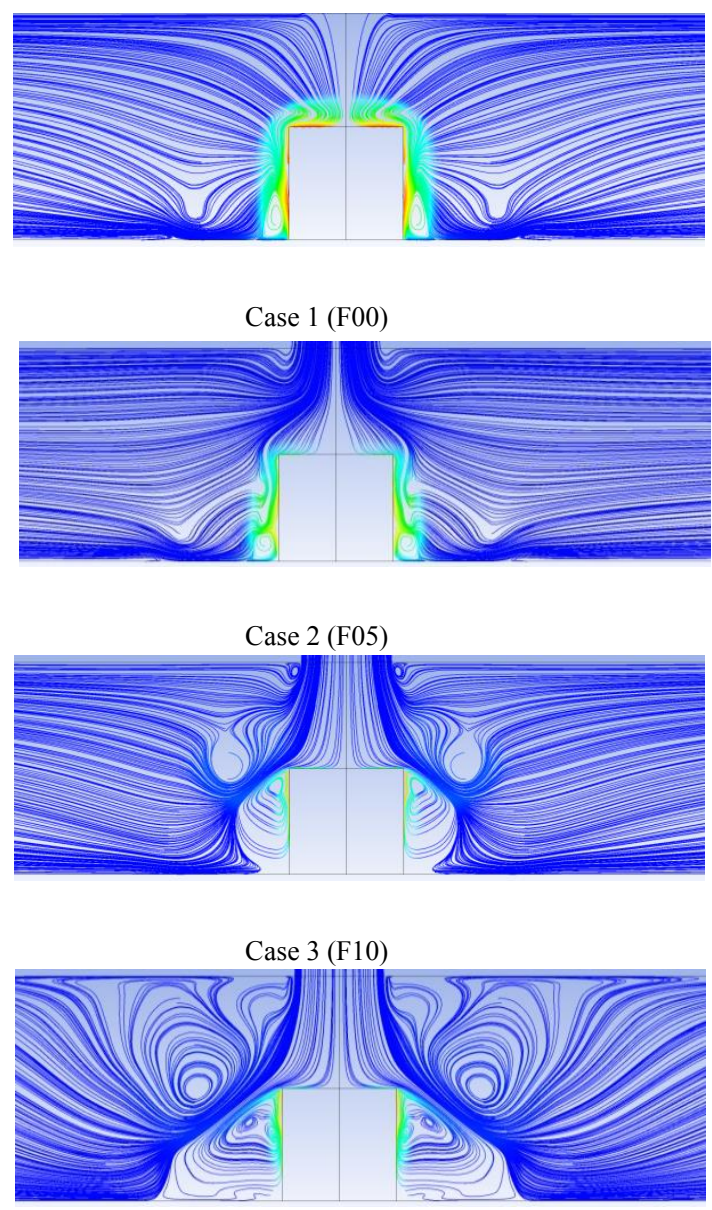

Case 4 (F15)

Fig. 5 Streamlines in the $\mathrm{YZ}$ plane at $\mathrm{x} / \mathrm{h}=0$ for $\mathrm{ReH}=3410$, colored by the temperature.
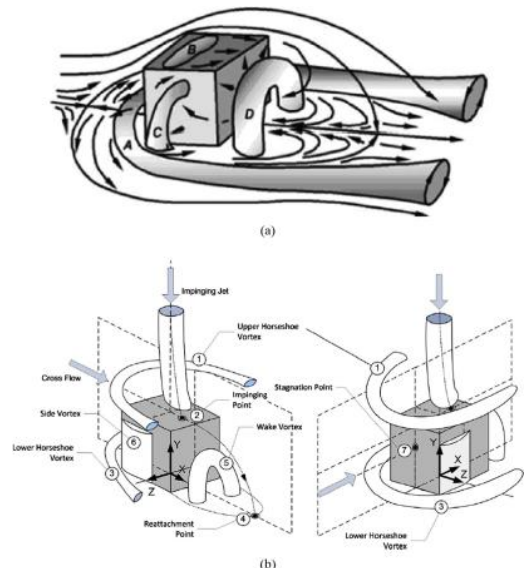

Fig. 6 (a) Mean flow structures around a wall mounted cube [10]. (b) Mean flow structures around a cube cooled by an impinging jet in a cross flow

\section{Nemdili Saleha, Nemdili Fadéla, Azzi Abbès}

As the flow configuration studied here is very complex and of great academic and application interest, many researchers $[10,6]$ worked to highlight the morphology of the nearest flow as showed at Figure 6. The two sketches correspond to cube without and with impingement jet respectively and highlight the main vortices accompanying this configuration. In Figure 7 an attempt to highlight the morphology of the flow is done by use of iso-surfaces of the so-called Q-criteria, function of vorticity and strain rate of the flow field. Iso-surfaces are colored by the velocity magnitude.

The horseshow vortex in front of the cube is well reproduced for the baseline case, as well as the upper horseshow vortex developing around the jet. This vortex is rapidly growing with Reynolds number ratio and becoming more complex and deviates down for the last case (F15).
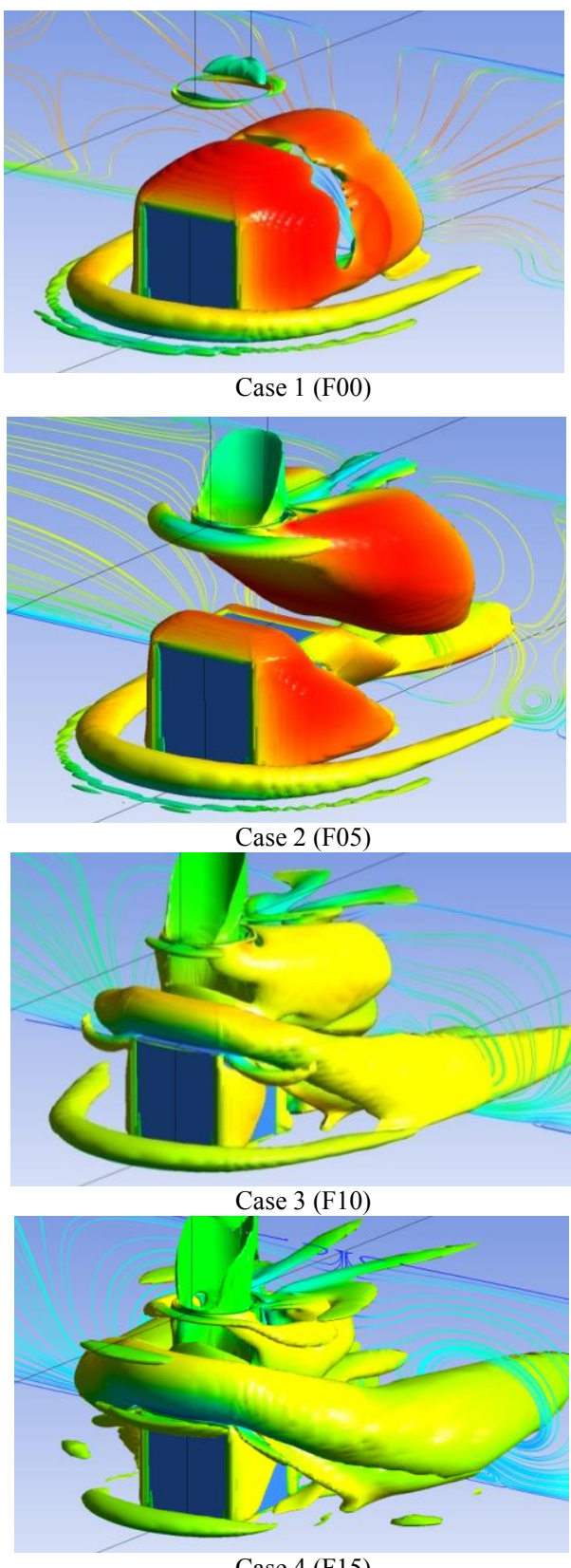

Case 4 (F15)

[6]. 


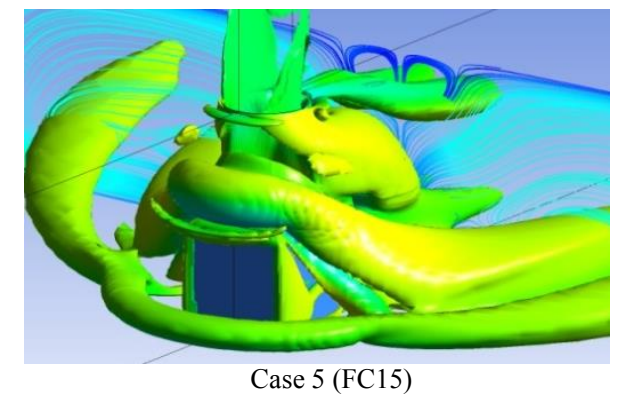

Fig. 7 Iso-surfaces of the so-called Q-criteria (6000 [ $\left.\left.\mathrm{s}^{\wedge}-2\right]\right)$ colored by the longitudinal velocity.

Before moving to the heat transfer fields, a quantitative comparison of longitudinal velocity at several locations before and after the cube is presented in Figure 9. The exact locations are highlighted by figure 8 and are as follow: one line in front of the cube (Line1 $\mathrm{x} / \mathrm{H}=-0.75$ ), one line on top of the cube (Line2, $\mathrm{x} / \mathrm{H}=0.0)$ and two lines downstream of the cube in the recirculation zone $(\mathrm{x} / \mathrm{H}=1$ and 1.5$)$. Globally, the longitudinal velocity profiles agree reasonably with the experimental ones in almost all locations. Some differences are reported in some profiles parts and can be attributed mainly to differences in inlet boundary conditions and also to the limitations of the turbulence modeling strategy adopted in frame of RANS approach. Nevertheless, the global behavior and qualitative results are reproduced in a satisfied level taking into account the numerically economic strategy adopted.

The three Reynolds numbers ratios as well as the base line case are presented. At the first location, one can see for the first case (baseline case) the slightly modified channel velocity profile. The profile is characterized by a decreasing velocity in the lowest half of the channel and an accelerating behavior in the top half. This is due mainly by the presence of the cube as an obstacle in the bottom part of the channel. This trend is conserved for the lowest Reynolds number ratio (0.5) but radically modified for the two remaining cases. As an effect of the negative jet penetration, the longitudinal velocity decreases even in the top half of the channel and for the highest Reynolds number ratio, one can see that the flow goes in negative direction in the middle of the channel.

When looking at the second location, which is located exactly on the middle top of the cube, the base line case is characterized by a normal channel profile with a small reverse flow near the top face of the cube. This reverse flow corresponds to the detached and reattached boundary layer on the top of the cube. This behavior is well predicted by the model especially the reverse flow for case 3 . Nevertheless, the highest Reynolds number ratio case (case 4) is worthy reproduced and the reverse flow seems to be completely ignored by the computation. This can be expected, since the two equation turbulence model is well known to reproduces poorly the stagnation regions. The next locations $(x / H=1$ and
1.5) highlights the jet deviation and penetration in the middle of the channel. The qualitative experimental behavior is satisfactory reproduced by the numerical model, while quantitatively some discrepancies can be reported. The vertical jet, after impinging the cube, goes in horizontal direction with a sensible acceleration in the middle of the channel. Note that for the modified case (case 5) and due to the chamfer the jet is deviated towards the bottom wall, which is well predicted by the numerical model.

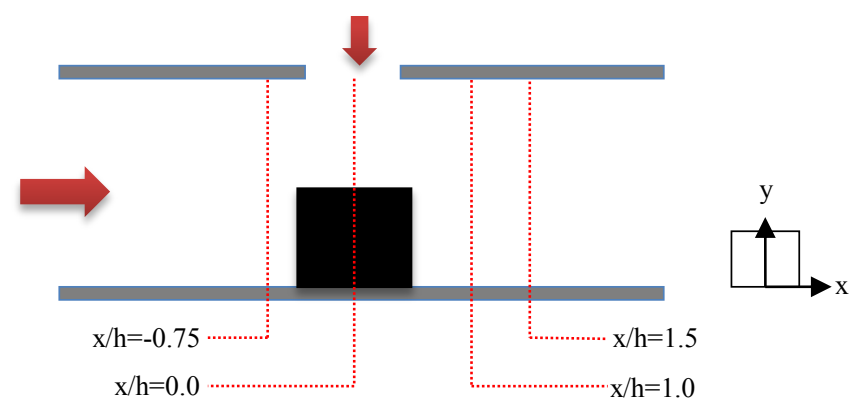

Fig. 8 Selected vertical lines where results are compared to experimental
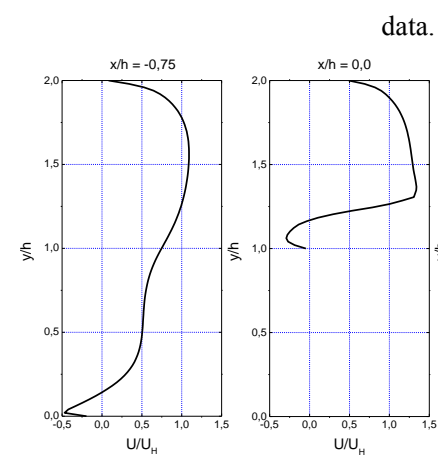
data.
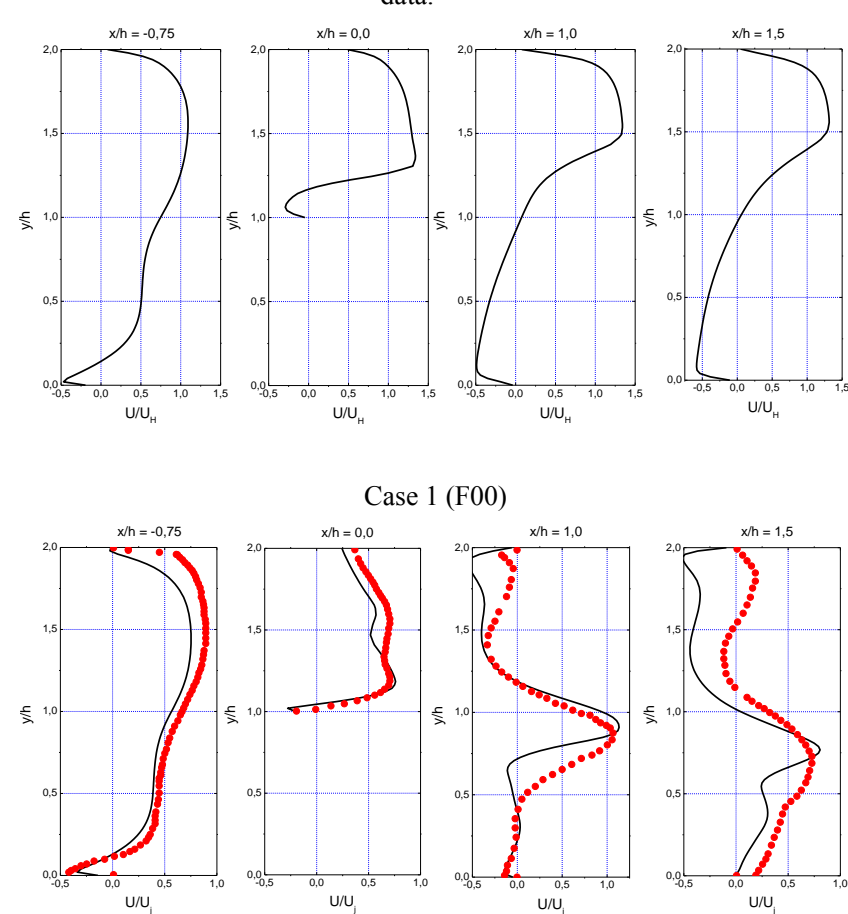

Case 2 (F05)
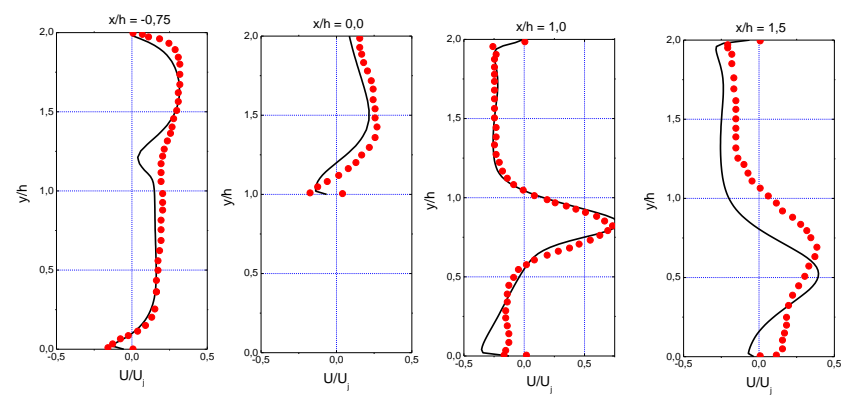

Case 3 (F10) 

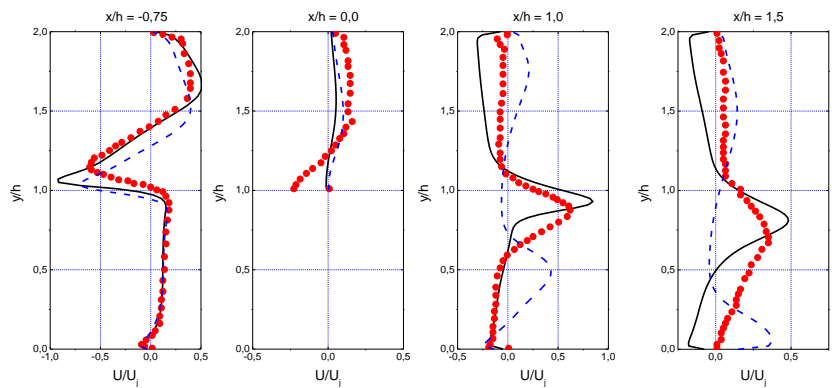

Case4(F15) and Case 5 (FC15)

— Present computation (regular cube)

- Experiment [06]

- - Present computation (cube with chamfer)

Fig. $9 U$ Velocity profiles $z / h=0$ for $R e_{H}=3410$.

Contours of temperature on the central plan $(\mathrm{z} / \mathrm{H}=0)$ are presented on Figure 10. As expected by previous results, the thickness of the thermal boundary layer decreases when increasing the Reynolds number ratio and the thermal shape follows the flow vortices. The wall heat flux at the surface of the cube is presented at figure 11 in a front view. At the front face, the contours have slightly the same behavior, while at the top and side faces the impinging jet power modifies and increases the wall heat distribution.
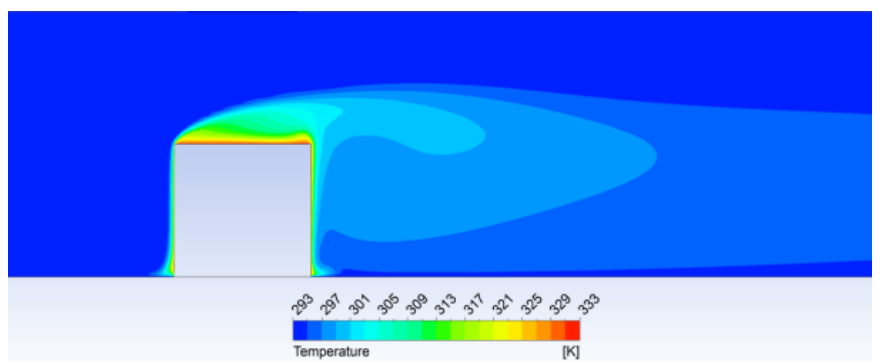

Case 1 (F00)

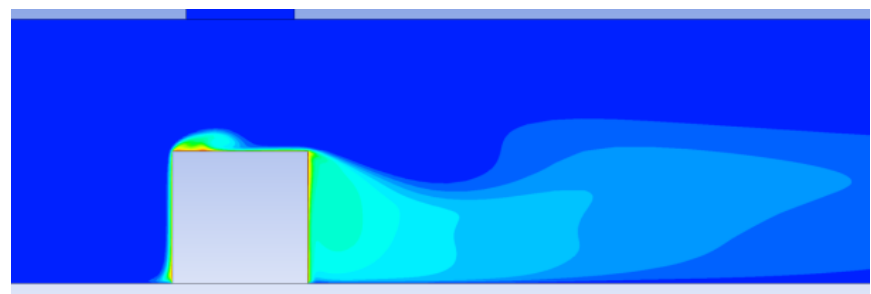

Case 2 (F05)

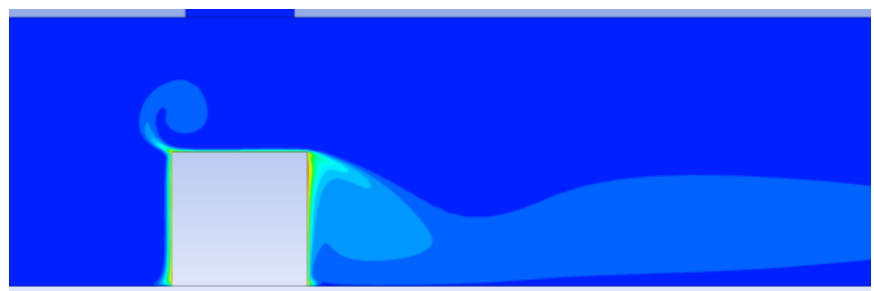

Case 3 (F10)

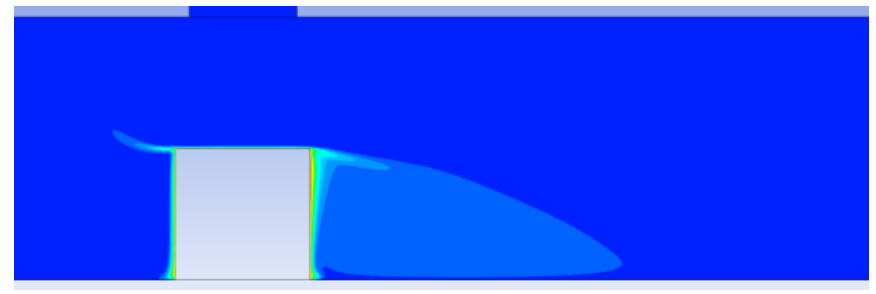

Case 4 (F15)

Fig. 10 Temperature contours at $\mathrm{z} / \mathrm{h}=0$ for $\mathrm{ReH}=3410$

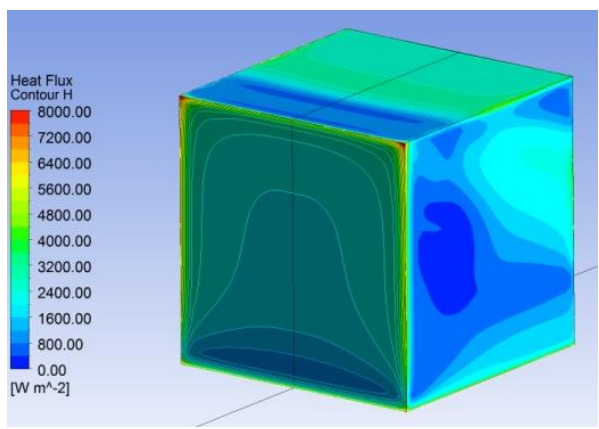

Case 1 (F00)

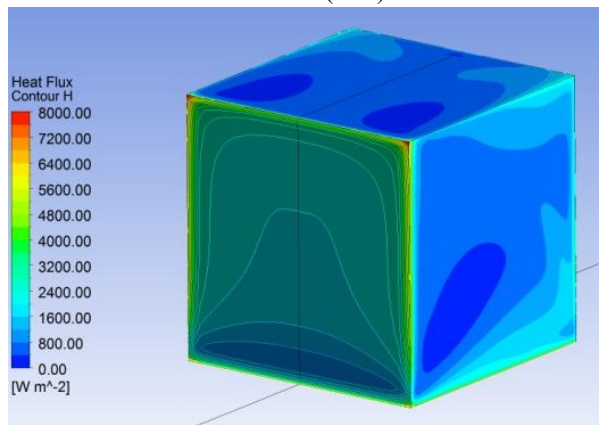

Case 2 (F05)

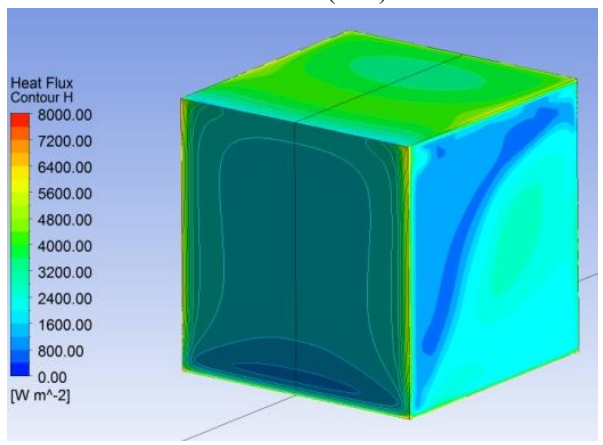

Case 3 (F10)

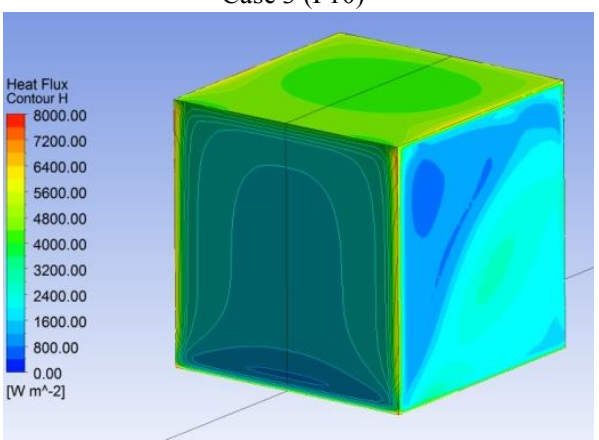

Case 4 (F15)

Fig. 11 Contours of Heat flux density on the surface of the cube, front view. 
Finally, the amount of wall heat flux exchanged by each side and the global heat flux are presented at Figure 12. The Figure shows that the wall heat flux at the front face is always the same for all configurations. It increases at side walls until $(\mathrm{Rej} / \mathrm{ReH}=1.0)$ and then remains constant. At rear side, it decreases from $(\mathrm{Rej} / \mathrm{ReH}=0.0)$ to $(\mathrm{Rej} / \mathrm{ReH}=0.5)$ and follows the behavior of the side faces. At the top face, the wall heat flux increases significantly when the impinging jet velocity increases. When looking at the global wall heat flux for the cube, it increases proportionally to the Reynolds number ratio.

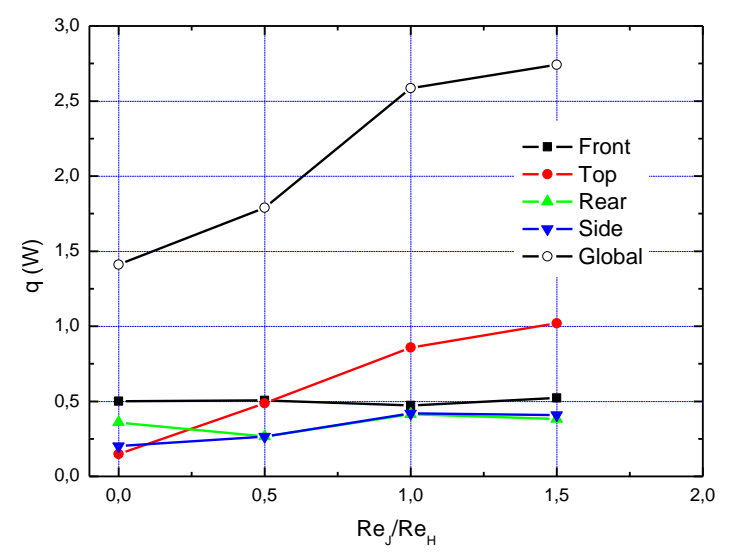

Fig. 12 Total heat flux on the global surface of the cube and by face

\subsection{Cube with chamfrain}

As previously mentioned, in order to increase cooling efficiency, the geometry of the cube is modified by adding a chamfer on its upper face. All cases studied here are at the same ratio of Reynolds numbers $(\mathrm{Rej} / \mathrm{ReH}=1.5)$ and as shown in Figure 2, five cases are considered. As a reminder; the test cases are noted: F15, FC15, FF15, FR15 and FS15, for the regular cube, the cube with complete chamfer, the cube with chamfer on the front face, the cube with chamfer on the back side and the cube with chamfer on the two lateral faces respectively. Figure 13 shows the streamlines in the $X Y$ plane at $z / h=0$ for the five tested cases. As the case (FS15) has only chamfer on the side faces and nothing on the front and rear faces, the configuration of the longitudinal flow is almost identical to that of the regular cube. On the other hand, for the cases FF15 and FR15 which correspond respectively to the front and rear chamfers, it can be seen that the jet decelerates at the top of the cube and then rotates sharply to form a radial wall jet. At the back, the flow separates and is attached downstream by creating a recirculation bubble while the front panel, it sweeps closely along the front face. This behavior can be explained by the fact that the main transverse flow pushes the jet against the front face. This change in the flow structure should lead to a drastic improvement of the cube cooling process. The last case (FC15), which is a cube with the chamfer on all four sides, shows almost the same change near the back face, while on the front face the flow pattern appears to be unchanged from the regular cube.

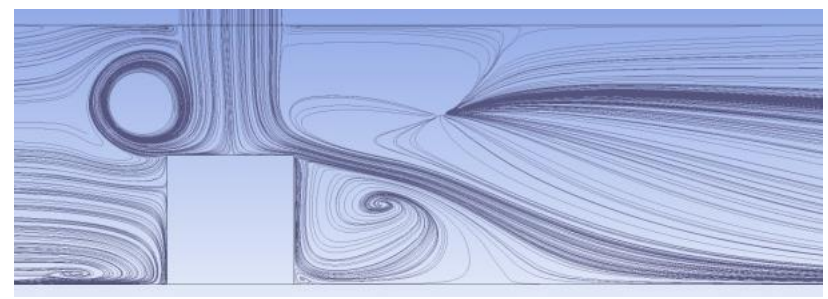

Regular cube (F15)

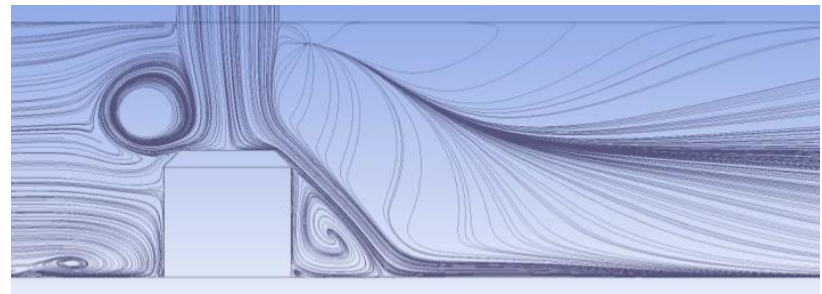

Full chamfer, FC15

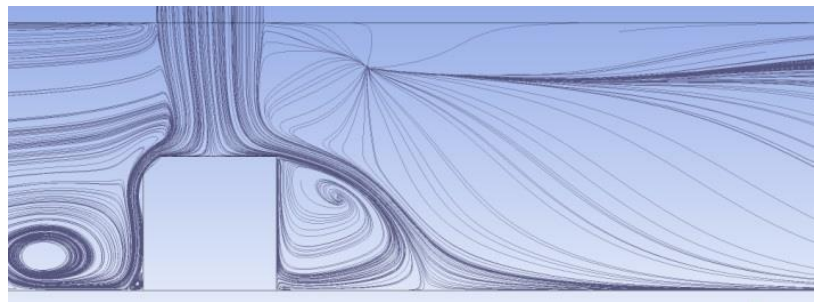

Chamfer only in the front face, FF15

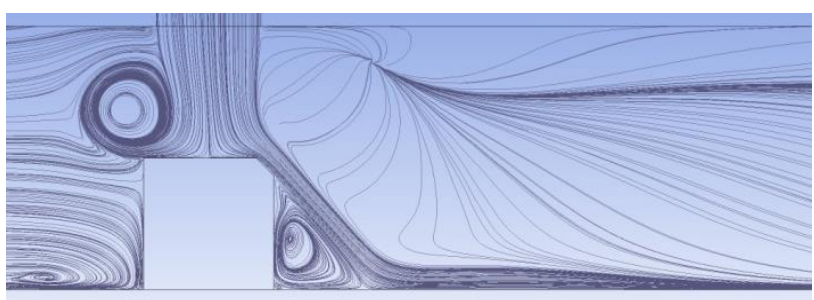

Rear face, FR15

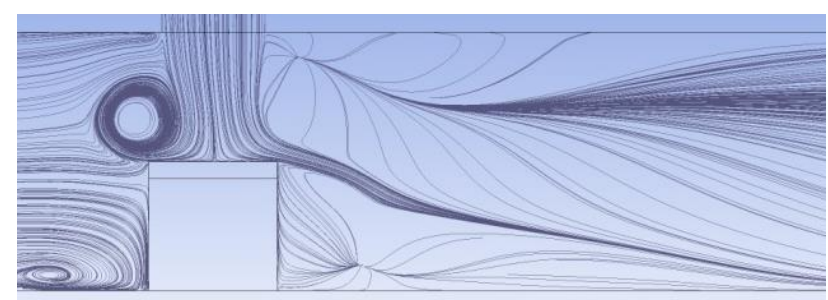

Side faces, FS15

Fig. 13 Streamlines in the $X Y$ plane at $z / h=0$ for $R e_{H}=3410$ and $\operatorname{Re}_{j} / \operatorname{Re}_{H}=1.5$. 
Figure 14 shows the overall heat flux for the five cases tested, while Figure 15 shows the amount of wall heat flux exchanged by each cube face. As expected by the flow configuration (Figure 13), it is confirmed that the cube with the chamfer on its front face ( FF15) produces the optimum cooling improvement. The cube with the chamfer on its rear face (FR15) comes in the second place followed by the case with the side chamfers (FS15) and at the last position the cube modified with chamfers on all four sides $(F C 15)$.

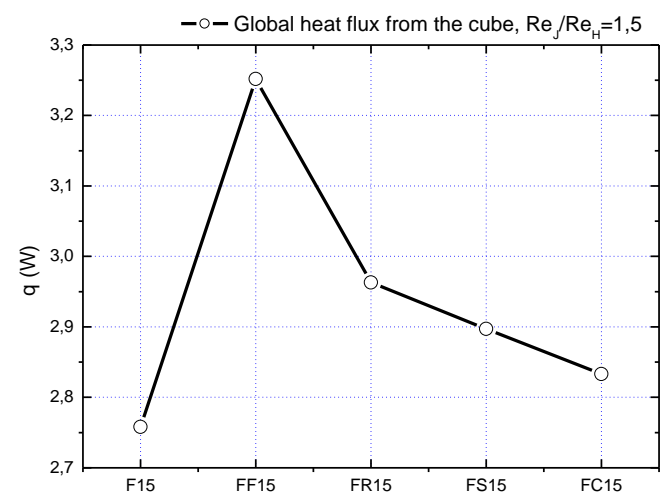

Fig. 14 Total heat flux on the global surface of the cube.

Considering each face separately, we can see that the cube FF15 produces the best performance on the upper, back and front sides. The side faces are better cooled in the cube FS15, while the cube $F F 15$ comes in second position. In all cases, the upper face produces the most important part of the wall heat flux. This is in agreement with the fact that the cold jet impacts directly on the upper face. The front face, which is exposed to the main transverse flow, gives the second most important part, in particular for the cube FF15 where the impact jet sweeps very closely the front face.

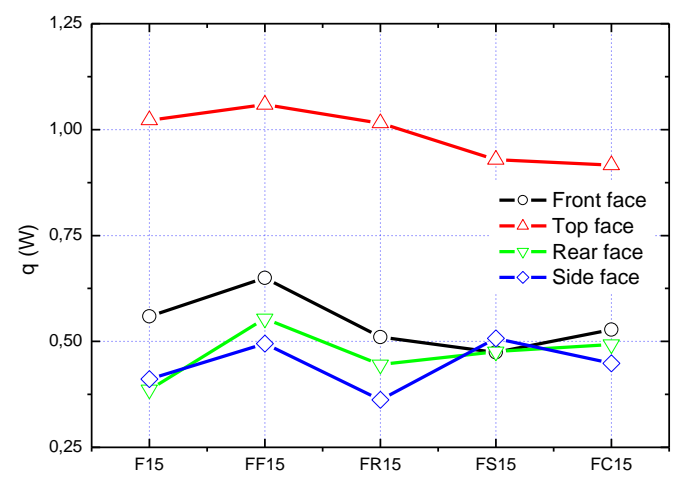

Fig.15 Amount of heat flux by face for the modified cases

Figure 16 shows a comparison of the streamlines, colored by the temperature, in the $Y Z$ plane at $x / h=0$. In all cases, the streamlines clearly show the different vortex structures near and far from the side faces of the cube. Comparing the two cases FR15 and F15, which respectively correspond to a rear face chamfer and to a regular cube (without chamfer), and as these two cases have no chamfer on the lateral faces, we can see that the configuration of longitudinal flow for these two cases is almost the same. At the corner of the cube, the flow separates and is attached downstream by creating a recirculation bubble that has a significant effect on the heat flow of the wall. On the other hand, one observes the same thing for the two cases FC15 and FS15 corresponding to a cube with chamfer on the four faces and a cube with chamfer only on the lateral faces. As expected from the flow configuration, the size of the two counter-rotating vortices for the FR15 case is higher compared to the two vortices developed in the case FS15.

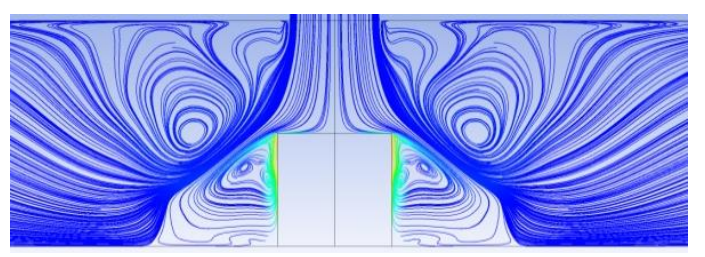

Regular cube (F15)
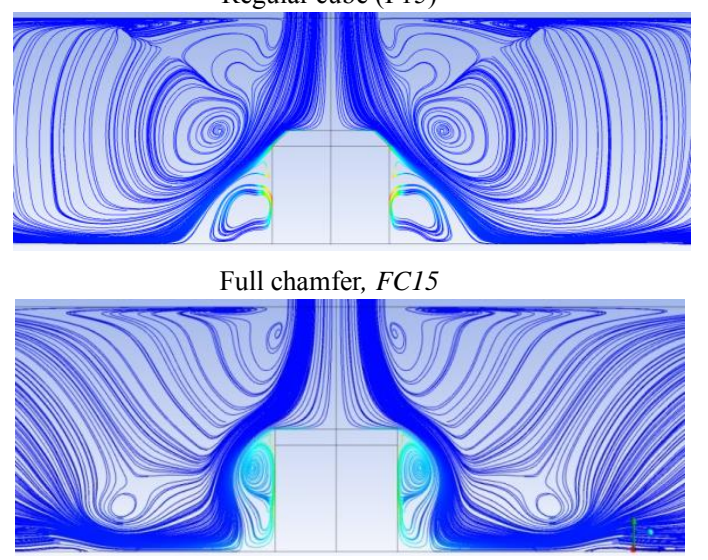

Chamfer only in the front face, FF15
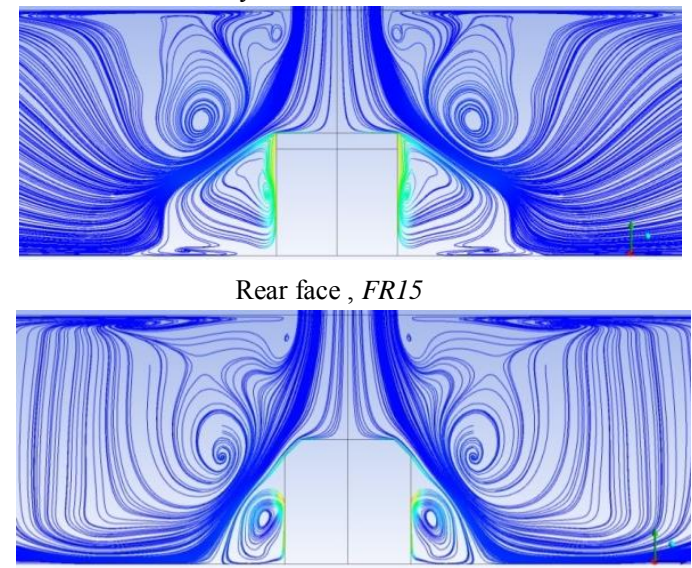

Side faces, FS15

Fig. 16 Streamlines in the $Y Z$ plane at $x / h=0$ for $R e_{H}=3410$, colored by the temperature

Figure 17 shows a three-dimensional view of the flow morphology using the iso-surfaces of the so-called Q-criteria, which correspond to the function of vorticity and strain rate of 
the flow field. The iso-surfaces are colored by the velocity magnitude. The figure shows the creation of two horseshoe vortexes, one around the jet and a second one around the cube. The two 'horseshoe' vortexes are well developed for the following cases: regular cube, cube with chamfer on side faces (FS15), cube with chamfer on back side (FR15) and cube with full chamfer. While in the case of the cube with the chamfer on the front $(F F 15)$ there is only one horseshoe vortex around the cube.
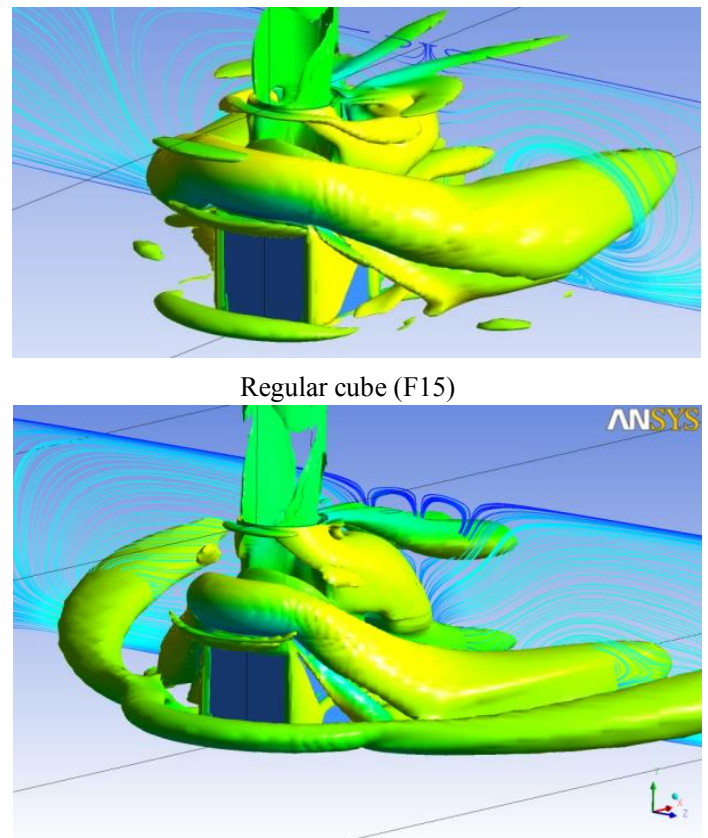

Full chamfer, FC15

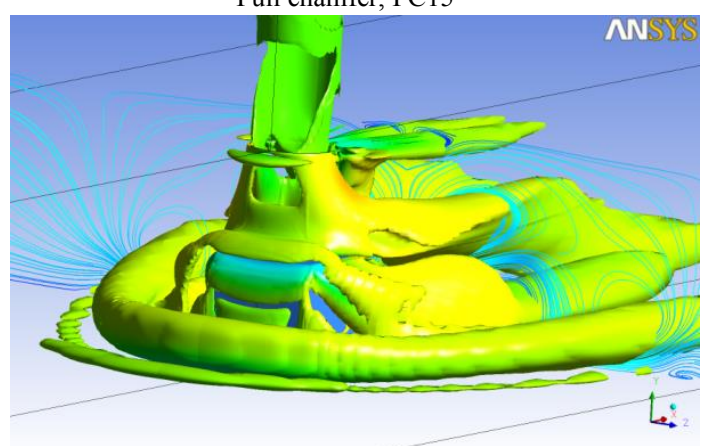

Chamfer only in the front face, FF15

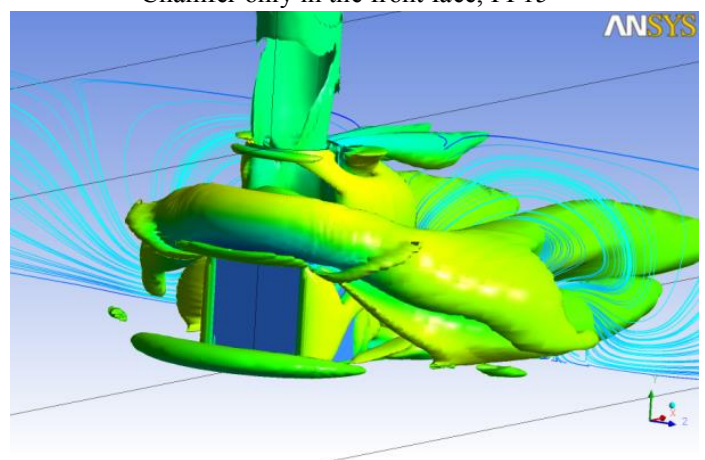

Rear face, FR15

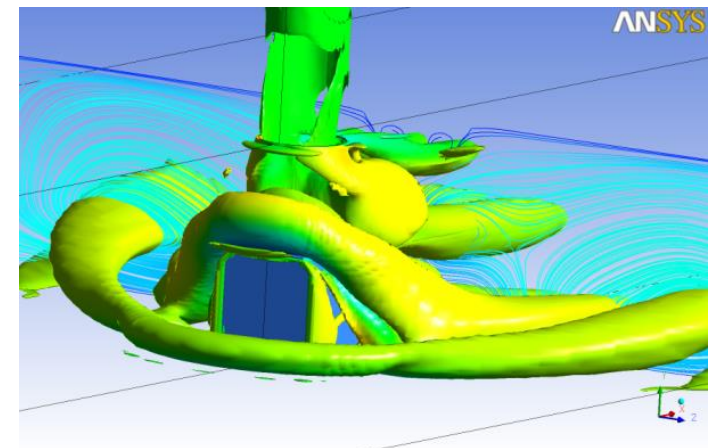

Side faces, FS15

Fig.17 Iso-surfaces of the so-called Q-criteria $\left(6000\left[\mathrm{~s}^{\wedge}-2\right]\right)$ colored by the longitudinal velocity

Figure 18 shows a quantitative comparison of the longitudinal velocity profiles $U$ in the $z / h=0$ plane, for the five cases $(F 15, F C 15, F F 15, F R 15, F S 15)$ at several longitudinal stations. As previously mentioned, the numerical results obtained for the $F 15$ and $F C 15$ cases were validated to the experimental results of Masip et al. [6]. Longitudinal velocity profiles were found to be reasonably consistent with experimental profiles in almost all stations.

At the first station $(x / h=-0.75)$, we can see for the cube with front chamfrain $(F F 15)$, where the impact jet sweeps very close to the front face, a speed profile slightly modified channel velocity profile compared to the basic case. The profile is characterized by a decreasing velocity in the lower half of the channel and an accelerated behavior in the upper half. This is mainly due to the presence of the cube as obstacle in the lower part of the channel. Whereas for the other cases (FC15, FR15, FS15), the velocity profile of the channel seems to be unchanged compared to the regular cube. As an effect of the jet penetration, the longitudinal velocity decreases even in the upper half of the channel. We can also distinguish the development of a negative flow in the middle of the channel.

At the second location $(x / h=0.0)$, which is located exactly in the middle of the cube, the figures are characterized by a normal channel velocity profile with a small reverse flow near the top face of the cube. This reverse flow corresponds to the detached and reattached boundary layer on the top of the cube. For the cube with chamfer $(F C 15)$, the reverse flow seems to be completely minimized by calculations.

At the next stations $(x / h=1$ and 1.5), the deviation of the jet and the penetration in the middle of the channel are well reproduced by the calculations. As noted above, the experimental behavior is reproduced very satisfactorily in its qualitative part (FC15 and FR15), whereas quantitatively some discrepancies can be reported. For these two cases, and due to the presence of the chamfer on the rear face, the jet is deflected towards the bottom wall, which is well predicted by the numerical model. Considering the cases (FF15 and FS15), the 
vertical jet, after impinging the cube, goes in the horizontal direction with a sensitive acceleration in the middle of the channel which is slightly similar to the experimental profile of the channel velocity profile.
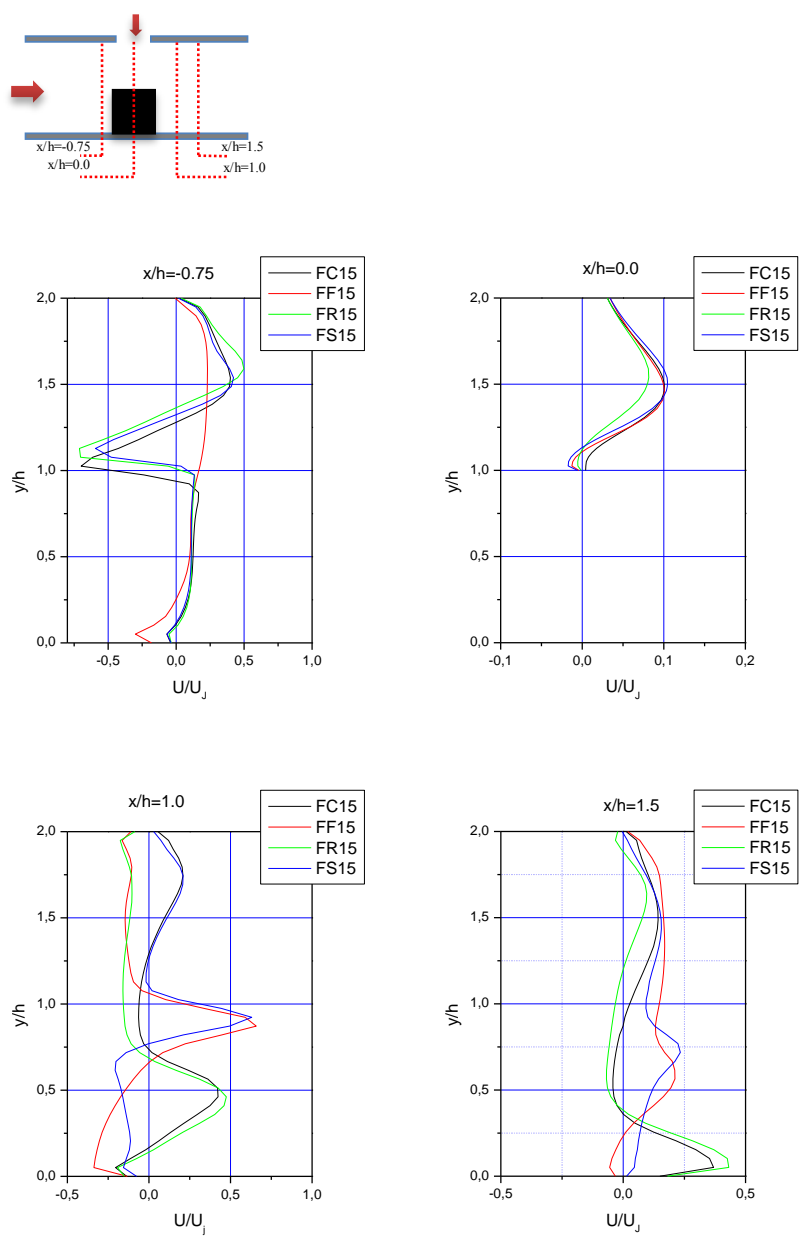

Fig. $18 U$ Velocity profiles $z / h=0$ for $R e_{H}=3410$.

Figure 19 shows the contours of the temperature on the central plane $(z / h=0)$ of the cube. In accordance with the dynamic field shown above, the structure of the thermal field follows the flow vortices. For the FR15 cube, it is observed that the highest values of the temperature appear near the rear wall of the cube. For the cube FF15, we note that the chamfer in front of the cube contributes to bring more cooling jet towards the front face of the cube. This aspect of the dynamic and thermal field contributes to better cooling the cube compared to the other configurations studied.

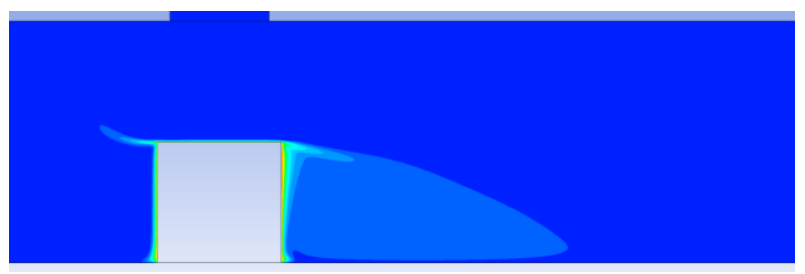

Regular cube (F15)

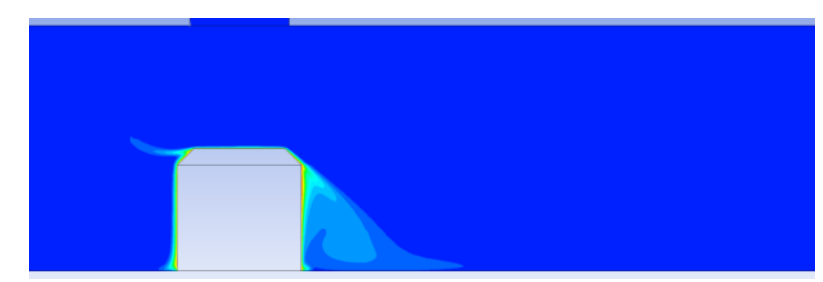

Full chamfer, FC15
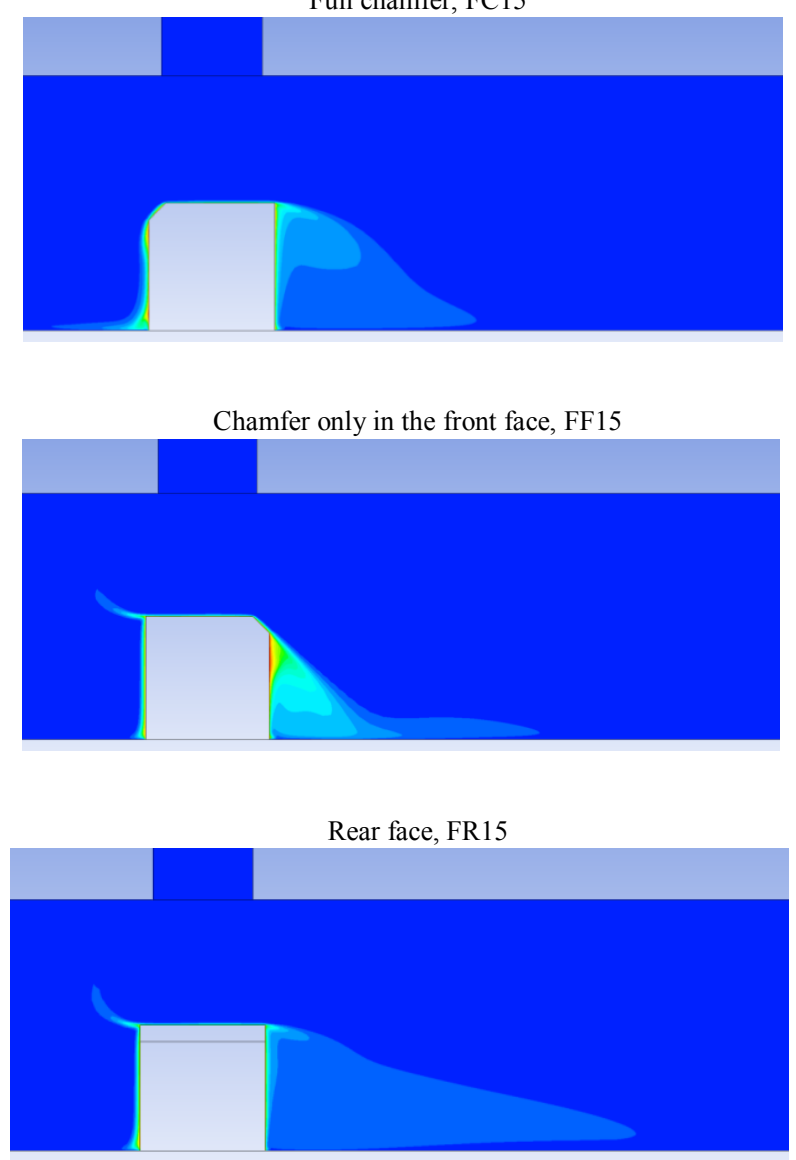

Side faces, FS15

Fig. 19 Temperature contours at $z / h=0$ for $R e_{H}=3410$

Finally, Figure 20 represents the density of the wall heat flux on the cube faces. For all cubes studied, the upper face, which is exposed to the impacting jet, the density of heat flux is the most intense. On the front face and for the four cases (F15, FC15, FR15 and FS15), the contours of the density of the heat flux is almost the same. However, the cube FF15, with the chamfer on its front face, the density of the heat flow is much more intense. These observations confirm the superiority of the FF15 cube compared to others. 


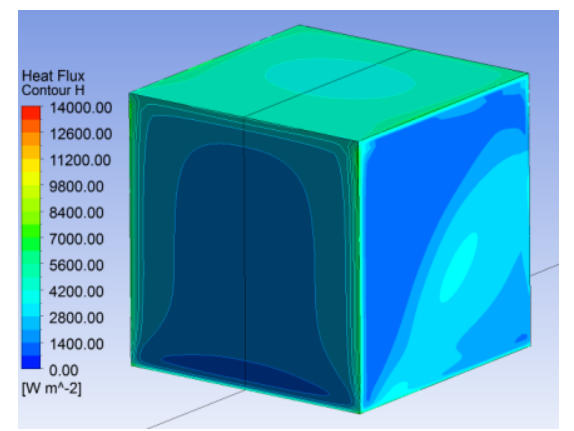

Regular cube (F15)

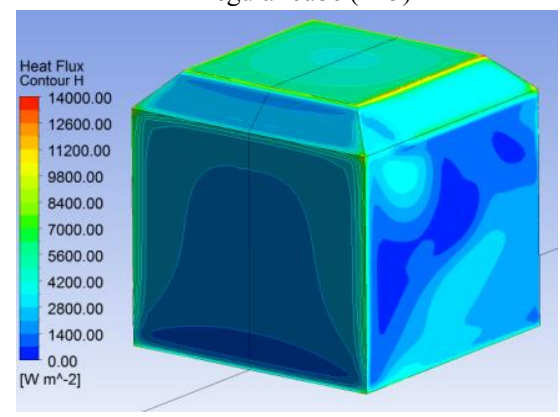

Full chamfer, FC15

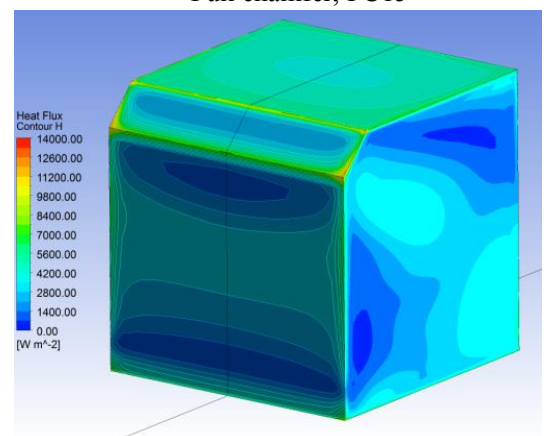

Chamfer only in the front face, FF15

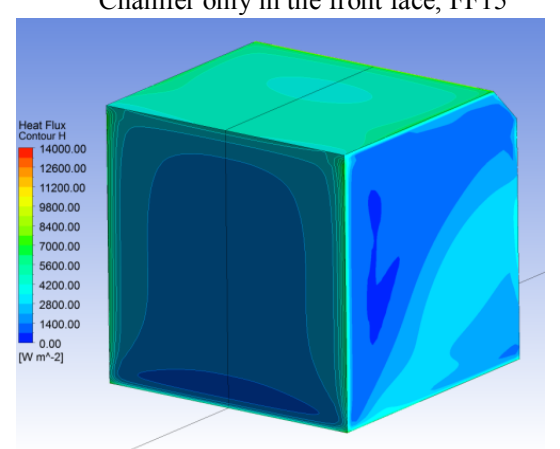

Rear face, FR15

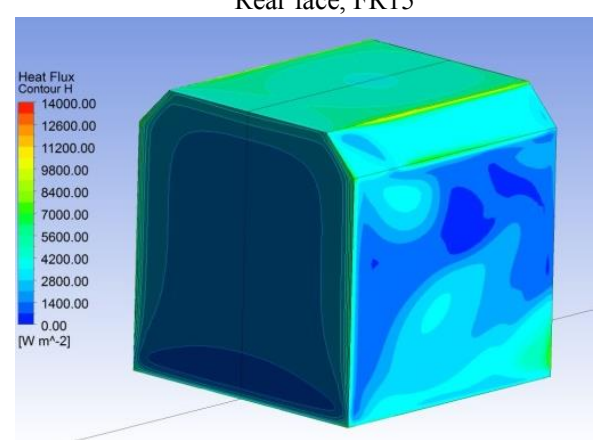

Side faces, FS15

Fig. 20 Contours of Heat flux density on the surface of the cube, front view.

\section{Conclusion}

Mean velocity and the thermal field of an impinging turbulent jet in cross-flow on a heated wall-mounted cube are numerically reproduced. In an attempt to improve the cooling efficiency, the cube is modified in several ways by adding a chamfer on its sharp limits. The present investigations focus on five cases, F15, FC15, FF15, FR15 and FS15, corresponding to: the regular cube, the cube with full chamfer, the cube with chamfer on its front face, the rear face and at both side faces respectively. The numerical results show that a small geometrical modification on the top cube face can increase the global wall heat flux and add some effectiveness to the cooling process. The results obtained show that the cube with chamfer between the front face and the upper face is the one that brings the best improvement compared to the other cases studied. Indeed, this modification allows the impinging jet to perfectly sweep the front face of the cube. The transverse flow intensifies this phenomenon by pushing the cold jet against the front face of the cube, which leads to a significant and consistent improvement in overall cooling.

\section{HHHQFH}

[1]. Tummers, M.J., Flikweert, M.A., Hanjalic' , K., Rodink, R., Moshfegh, B., 2005. Impinging jet cooling of wallmounted cubes. In: Proc. of ERCOFTAC, International Symposium on Engineering Turbulence Modelling and Experiments - ETMM6, Sardinia, Italy. pp. 773-791.

[2]. Rundström, D., Moshfegh, B., 2006a. Investigation of flow and heat transfer of an impinging jet in a cross-flow for cooling of a heated cube. ASME J. Electronic Pack. $128,150-156$.

[3]. Rundström, D., Moshfegh, B., 2009. Large-eddy simulation of an impinging jet in a cross-flow on a heated wall-mounted cube. Int. J. Heat Mass Transfer 52, 921931.

[4]. M. Popovac *, K. Hanjalic, 'Large-eddy simulations of flow over a jet-impinged wall-mounted cube in a cross stream', International Journal of Heat and Fluid Flow 28 (2007) 1360-1378

[5]. M. Popovac 1, K. Hanjalic, 'Vortices and heat flux around a wall-mounted cube cooled simultaneously by a jet and a crossflow', International Journal of Heat and Mass Transfer 52 (2009) 4047-4062

[6]. Yunesky Masip, Alejandro Rivas, Gorka S. Larraona, Raúl Anton, Juan Carlos Ramos and Bahram Moshfegh, 'Experimental study of the turbulent flow around a single wall-mounted cube exposed to a cross-flow and an impinging jet', International Journal of Heat and Fluid Flow, 38 (2012), pp. 50-71

[7]. B.P. Leonard, the ULTIMATE conservative difference scheme applied on unsteady one-dimensional advection, Comput. Methods Appl. Mech. Eng. 88 (1991) 17-74.

[8]. F. R. Menter, M. Kuntz and R. Langtry, Ten years of Industrial Experience with the SST turbulence model, 
Turbulence, Heat and Mass Transfer IV, Antalya, Turkey, 12-17 October, 2003.

[9]. M. Flikweert, Flow and heat transfer investigation of wallmounted cubes in cross-flow, M.Sc. thesis, Delft University of Technology, Delft, The Netherlands, 2005.

[10]. Martinuzzi, R.J., Tropea, C., 1993. The flow around surface mounted, prismatic obstacles placed in a fully developed channel flow. J. Fluids Eng. 115, 85-92.

[11]. Nemdili Saleha, Nemdili Fadèla, Azzi Abbès, Improving cooling effectiveness by use of chamfers on the top of electronic components, Microelectronics Reliability, 55 (2015) 1067-1076. (2015)

\section{Creative Commons Attribution License 4.0 (Attribution 4.0 International, CC BY 4.0)}

This article is published under the terms of the Creative Commons Attribution License 4.0

https://creativecommons.org/licenses/by/4.0/deed.en_US 